\section{Avaliação dos sistemas de informações sobre nascidos vivos e óbitos no Brasil na década de 2000}

\author{
Evaluation of information systems on live births \\ and mortality in Brazil in the 2000s
}

\section{Evaluación de los sistemas de información sobre nacimientos y muertes en Brasil en la década}

de 2000

\footnotetext{
1 Instituto de Medicina Integral de Pernambuco Prof. Fernando Figueira, Recife, Brasil.

2 Universidade Federal de Pernambuco, Recife, Brasil. 3 Instituto de Comunicação e Informação Científica e Tecnológica em Saúde, Fundação Oswaldo Cruz, Rio de Janeiro, Brasil.

Correspondência P. G. Frias Instituto de Medicina Integral de Pernambuco Prof. Fernando Figueira. Rua dos Coelhos $s / n$, Recife, PE 50070-590, Brasil. pfrias@imip.org.br
}

\begin{abstract}
The Brazilian Ministry of Health's mortality and live birth information systems (SIM and SINASC) are valuable tools for measuring population health status. The purpose of this study was to assess the adequacy of vital information systems from 1999-2001 to 2008-2010 in the States and major geographic regions of Brazil. Indicators were constructed to assess data coverage, regularity, and quality for SIM and SINASC. The municipalities (counties) were divided into three categories according to adequacy of data on deaths and live births by calculating an adequacy index based on the proportion of the population living in each category. The adequacy index for Brazil increased from 73.9 to 89.1 in the SIM and from 80.5 to 90.9 in the SINASC from the first to the second triennium, respectively. Improvements occurred in all the indicators, in all regions of the country, and in most States. Analysis of the adequacy of vital information in Brazil showed clear improvement throughout the country, particularly in States of the North and Northeast regions.
\end{abstract}

Information Systems; Vital Statistics; Health Evaluation; Live Births
Paulo Germano de Frias 1,2 Célia Landmann Szwarcwald 3 Pedro Israel Cabral de Lira ${ }^{2}$

\section{Resumo}

Os sistemas de informações sobre mortalidade (SIM) e nascidos vivos (SINASC) do Ministério da Saúde constituem instrumentos de aferição da situação de saúde. O objetivo deste artigo é avaliar o nível de adequação dos sistemas de informações vitais entre os triênios 1999-2001 e 2008-2010 nas Unidades da Federação (UF) e regiões brasileiras. Com base nos dados do SIM e SINASC, foram construídos indicadores para avaliar a cobertura, a regularidade e a qualidade das informações. Os municípios foram classificados em três categorias, de acordo com a adequação de óbitos e nascidos vivos, calculando-se um índice de adequação baseado na proporção da população residente em cada uma das categorias. O Índice de Adequação para o Brasil aumentou de 73,9 para 89,1 (SIM) e de 80,5 para 90,9 (SINASC), entre os dois triênios, respectivamente. Os avanços ocorreram em todos os indicadores, em todas as regiões e grande parte das UF. A análise do nível de adequação das informações vitais no Brasil demonstrou inequívoca melhoria da situação em todo o país, particularmente nas UF das regiões Norte e Nordeste.

Sistemas de Informação; Estatísticas Vitais; Avaliação em Saúde; Nascimento Vivo 


\section{Introdução}

Com a proximidade de 2015, ano de referência para a avaliação dos compromissos assumidos pelos 189 países da Organização das Nações Unidas quanto às Metas de Desenvolvimento do Milênio, as estimativas da mortalidade infantil têm despertado interesse crescente 1 .

Diferentemente das nações desenvolvidas, os países em desenvolvimento não dispõem, em geral, de sistemas de informação de registros contínuos ou, se os têm, são usualmente incompletos e não geram dados suficientes para mensurar os indicadores de mortalidade em âmbito nacional, com fidedignidade 2,3,4.

Indicadores construídos com base em sistemas de informações nacionais de uso rotineiro, que apresentam coberturas incompletas, podem induzir a interpretações incorretas da magnitude e das tendências espaço-temporais ${ }^{3}$. No que diz respeito à mortalidade adulta, tendências distintas das taxas de mortalidade por doenças cardiovasculares foram encontradas, antes e após a correção dos dados de mortalidade 5 . A elevada proporção de óbitos sem definição da causa básica, em alguns estados brasileiros, afeta, igualmente, a magnitude das taxas de mortalidade específicas por causas 6 .

Já no caso da mortalidade infantil, diferenças regionais na notificação dos eventos vitais podem provocar subestimação das taxas, justamente nas áreas de pior nível socioeconômico e maiores dificuldades de acesso ao sistema de saúde 7 . Por outro lado, aumentos na cobertura dos sistemas de informações, ao longo do tempo, podem afetar a evolução temporal das estimativas de mortalidade infantil, sob a suposição de sub-registro constante no tempo ${ }^{8}$.

Para os países que têm problemas nas informações vitais de registro contínuo, são aplicados inquéritos populacionais com questões dirigidas às mães sobre o número de filhos nascidos vivos e os sobreviventes 9,10. Esses métodos traçam um diagnóstico momentâneo e parcial, incapaz de apreender as mudanças em curso 11.

Além das limitações dos inquéritos domiciliares para estimação da mortalidade infantil, como bem documentado anteriormente 11,12, a aplicação de métodos distintos de análise dos dados pelos próprios países ou por organismos internacionais tem produzido, frequentemente, resultados discordantes 7 . A diferença obtida nas mensurações dos indicadores que se valem de múltiplas abordagens metodológicas pode gerar dúvidas quanto aos resultados da intervenção para o alcance das metas 13,14.

No Brasil, na década de 1990, inconsistências nas estimativas indiretas da mortalidade infantil e nas coberturas dos sistemas de informações do Ministério da Saúde foram encontradas 15. Mais recentemente, nos anos 2000, as estimativas da mortalidade infantil baseadas na Pesquisa $\mathrm{Na}$ cional por Amostra de Domicílios (PNAD) indicavam um patamar de estabilidade após 2005, enquanto as estimativas fundamentadas na correção das estatísticas vitais mostraram tendência contínua de decréscimo até 2009 8. Resultados do Censo Demográfico de 2010 confirmaram o decréscimo (Instituto Brasileiro de Geografia e Estatística. Censo Demográfico 2010. Resultados Gerais da Amostra. http://www.ibge.gov.br/ home/presidencia/noticias/imprensa/ppts / 00000008473104122012315727483985.pdf (acessado em 30/Jun/2013).

Diante das limitações dos sistemas de informações vitais, nas últimas duas décadas, o Ministério da Saúde realizou investimentos para a sua melhoria. Entre as estratégias adotadas estão o suporte normativo às ações do processo de produção e análise das informações vitais bem como a constituição de pactos envolvendo as três esferas de gestão - municipal, estadual e federal 4,16 . Iniciativas para reduzir a magnitude das causas mal definidas, pela vigilância das mortes sem definição da causa básica, dos óbitos maternos, fetais e infantis contribuíram, de igual forma, para a melhoria das informações vitais 17 , como se evidencia em manuais do Ministério da Saúde 18.

Com a corresponsabilização das informações nos três âmbitos governamentais, o papel do município na produção e processamento das informações foi enfatizado 4 . O Ministério da Saúde, por meio do Departamento de Informática do SUS (DATASUS; http://www.datasus.gov.br), passou a disponibilizar informações sobre óbitos e nascidos vivos, por município, com maior agilidade. E, para melhorar o acompanhamento do fluxo das informações, foi criado recentemente um painel de monitoramento dos dados sobre mortalidade disponível na Internet (http://svs. aids.gov.br/dashboard/mortalidade/infantil. show.mtw).

Paralelamente, iniciativas para avaliar os sistemas de informações vitais no país foram desenvolvidas, utilizando uma multiplicidade de abordagens metodológicas 19. Dentre elas, citam-se: o relacionamento e a comparação entre sistemas de informações; a análise da adequação das informações por meio de indicadores do próprio sistema 15 e a busca ativa de eventos vitais não informados ao Ministério da Saúde 20,21,22.

A esse cenário nacional agrega-se o intenso debate internacional em torno de metodologias apropriadas para avaliação dos objetivos do milênio, as fontes preferenciais para o cálculo dos indicadores e o estímulo ao desenvolvimento de 
metodologias para análise da cobertura dos sistemas de informações, utilizando dados do próprio sistema 2,3.

Iniciativas que apontem a situação subnacional quanto às estatísticas vitais produzidas pelos sistemas de informações do Ministério da Saúde podem contribuir com a discussão em curso 7 . Este trabalho tem como objetivo avaliar o nível de adequação dos sistemas de informações vitais entre os triênios 1999-2001 e 2008-2010, analisando as mudanças ocorridas nas Unidades da Federação (UF) e grandes regiões brasileiras por meio de indicadores municipais construídos com dados dos próprios sistemas de óbitos e nascidos vivos do Ministério da Saúde.

\section{Material e métodos}

Os dados sobre óbitos e nascidos vivos analisados foram provenientes do Sistema de Informações sobre Mortalidade (SIM) e Sistema de Informações sobre Nascidos Vivos (SINASC) referentes a todos os municípios brasileiros, para os triênios 1999-2001 e 2008-2010, disponíveis na página de Internet do DATASUS (http://www.datasus. gov.br). A opção de utilizar triênios teve como objetivo dar maior estabilidade aos indicadores municipais, considerando-se a média dos dados vitais informados a cada três anos, em virtude da grande proporção (46\%) de municípios com menos de 10 mil habitantes. Para análise do nível de adequação das informações vitais, o método descrito por Andrade \& Szwarcwald 23 foi adaptado.

Os indicadores usados para estabelecer os níveis de adequação das informações vitais dos municípios relacionados à mortalidade foram o Coeficiente Geral de Mortalidade Padronizado por Idade (CGMP), o Desvio Médio Relativo do Número de Óbitos (DMRO) e a proporção de óbitos com causas mal definidas no total de óbitos (percentual de óbitos mal definidos). Os dois primeiros indicadores representam a cobertura $\mathrm{e}$ a regularidade do sistema, respectivamente, e o terceiro, a qualidade das notificações dos óbitos no que se refere à causa básica.

Para calcular o CGMP municipal em 2000, foram utilizadas as populações censitárias dos municípios por faixa etária (menor de um ano; $1-4$; 5-9; 10-14; 15-19; 20-29; 30-39; 40-49; 50-59; 60-69; 70 e mais anos). Para o ano de 2009, foi necessário estimar as populações dos municípios por grupo de idade. As populações totais dos municípios e as por grupo etário foram obtidas por meio da interpolação das populações censitárias de 2000 e 2010 e as respectivas proporções por faixa de idade. Para os municípios que foram criados após 2000, foi utilizada a distribuição etá- ria de 2010. A população padrão para o cálculo do CGMP foi a brasileira correspondente aos anos de 2000 e 2009 (DATASUS. Informações de Saúde. http://tabnet.datasus.gov.br/cgi/popestim/ popdescr.htm, acessado em Jun/2013). Os CGMP foram calculados com a média dos óbitos por grupo de idade dos triênios 1999-2001 e 20082010 e as populações municipais por grupo de idade nos anos de 2000 e 2009.

Para o cálculo do DMRO nos triênios 19992001 e 2008-2010, utilizou-se a razão entre o desvio médio do número de óbitos (DMO) e a média de óbitos (M) no triênio, dado por:

$\mathrm{DMRO}=\mathrm{DMO} / \mathrm{M} \times 100 \%$, onde

$\left.\mathrm{DMO}=\left(\left|\mathrm{O}_{\mathrm{t}-1}-\mathrm{M}\right|+\mid \mathrm{O}_{\mathrm{t}}-\mathrm{M}\right)+\left|\mathrm{O}_{\mathrm{t}+1}-\mathrm{M}\right|\right) / 3$

$\mathrm{M}=\left(\mathrm{O}_{\mathrm{t}-1}+\mathrm{O}_{\mathrm{t}}+\mathrm{O}_{\mathrm{t}+1}\right) / 3$

$\mathrm{O}_{\mathrm{t}}=$ número de óbitos no ano $\mathrm{t}$.

O percentual de óbitos mal definidos foi obtido pela razão entre a soma de óbitos mal definidos e a soma do total de óbitos em cada triênio.

A classificação do município em relação ao nível de adequação das informações foi definida pelos valores extremos representados por percentis $10 \%$, para o indicador CGMP, e os percentis $90 \%$, para os DMRO e percentual de óbitos mal definidos, entre os municípios da UF, região e Brasil.

Para definição do nível de adequação das informações sobre nascidos vivos, foi utilizada a Razão entre Nascidos Vivos Informados e Estimados (RZNV) e o Desvio Médio Relativo do Número Total de Nascidos Vivos (DMRNV). Os indicadores expressam a cobertura e a regularidade das informações do sistema, respectivamente. Para calcular a RZNV municipal nos anos considerados, o número estimado de nascidos vivos foi obtido com base na população de menores de um ano de idade, após correção pela subenumeração, utilizando procedimento adotado no cálculo de tábuas de vida 24.

Para o cálculo do DMRNV nos triênios 19992001 e 2008-2010, utilizou-se a razão entre o desvio médio de nascidos vivos (DMNV) e o número médio de nascidos vivos $(\mathrm{N})$ no triênio, dado por: $\mathrm{DMRNV}=\mathrm{DMNV} / \mathrm{N} \times 100 \%$, onde

$\left.\mathrm{DMNV}=\left(\left|\mathrm{NV}_{\mathrm{t}-1}-\mathrm{N}\right|+\mid \mathrm{NV}_{\mathrm{t}}-\mathrm{N}\right)+\left|\mathrm{NV}_{\mathrm{t}+1}-\mathrm{N}\right|\right) / 3$ $\mathrm{N}=\left(\mathrm{NV}_{\mathrm{t}-1}+\mathrm{NV}_{\mathrm{t}}+\mathrm{NV}_{\mathrm{t}+1}\right) / 3$

$\mathrm{NV}_{\mathrm{t}}=$ número de nascidos vivos no ano $\mathrm{t}$.

A classificação do município em relação ao nível de adequação das informações foi definida pelos valores extremos representados pelo percentis $10 \%$, para a RZNV, e os percentis $90 \%$, para o DMRNV, considerando os valores dos indicadores nos municípios das UF, região e Brasil.

No caso dos indicadores de cobertura, os percentis $10 \%$ representam os valores extremos da distribuição dos indicadores entre os municípios. Acréscimos expressam os aumentos dos limites 
críticos inferiores durante a década. O mesmo pode ser dito em relação aos percentis $90 \%$ dos indicadores de irregularidade e percentual de óbitos mal definidos. Decréscimos expressam os avanços dos limites críticos superiores no período 2000-2009.

\section{Análise dos dados}

Para análise da adequação das informações sobre óbitos nos dois triênios estudados, os indicadores CGMP, o DMRO e o percentual de óbitos mal definidos foram categorizados em três níveis distintos:

- Categoria I: informações vitais consideradas adequadas. Definida por: CGMP $\geq 5,0$ por 1.000 habitantes e DMRO $<20 \%$ e percentual de óbitos mal definidos $<10 \%$;

- Categoria II: informações vitais não adequadas. Definida por: CGMP $\geq 3,0$ e CGMP $<5,0$ por 1.000 habitantes ou DMRO $\geq 20 \%$ ou percentual de óbitos mal definidos $\geq 10 \%$, isto é, pelo menos um dos indicadores não atinge o padrão adequado;

- Categoria III: informações vitais deficientes. Definida por: CGMP < 3,0 por 1.000 habitantes.

Para sumarizar a adequação das informações de óbitos por UF, região e totalidade do Brasil, foi construído o índice de adequação das informações sobre mortalidade, dado por:

Índice de adequação $=\left(\mathrm{P}_{1}+0,5 \times \mathrm{P}_{2}-\mathrm{P}_{3}\right) / \mathrm{P} \mathrm{x}$ $100 \%$, onde

$\mathrm{P}_{\mathrm{i}}=$ população residente dos municípios classificados na Categoria I, onde $\mathrm{I}=1,2$ ou 3 .

$\mathrm{P}=\mathrm{P}_{1}+\mathrm{P}_{2}+\mathrm{P}_{3}$.

Para a análise da adequação das informações sobre nascidos vivos nos dois triênios considerados, mediante os indicadores RZNV e o DMRNV, foram categorizados em três níveis:

- Categoria I: informações vitais consideradas adequadas. Definida por: RZNV $\geq 0,85$ e_DMRNV $<20 \%$;

- Categoria II: informações vitais não adequadas. Definida por: RZNV $\geq 0,60$ e RZNV $<0,85$ ou $\mathrm{DMRNV} \geq 20 \%$, isto é, quando pelo menos um dos indicadores não atinge o padrão adequado;

- Categoria III: informações vitais deficientes. Definida por: $\mathrm{RZNV}<0,60$.

De maneira análoga à análise dos dados de mortalidade, para sumarizar a adequação das informações de nascidos vivos por UF, região e totalidade do Brasil, foi construído o índice de adequação das informações sobre nascidos vivos, em que as categorias se referem às informações de nascidos vivos.

A comparação da adequação das informações sobre óbitos e nascidos vivos por UF e região, obtidas pelas informações dos municípios que a compõem, nos dois triênios correspondentes ao início e final da década, foi feita segundo o número de municípios e a proporção da população residente em cada uma das três categorias, e pelo índice de adequação.

\section{Resultados}

Na Tabela 1, estão dispostos os percentis $10 \%$ do CGMP e da RZNV, e os percentis $90 \%$ do DMRO, do DMRNV e do percentual de óbitos mal definidos segundo UF e região, para os anos de 2000 e 2009. Em quase todas as UF, os percentis $10 \%$ correspondentes aos indicadores CGMP e à RZNV apresentaram acréscimos expressivos, sobretudo no Norte e Nordeste. Na Região Nordeste, no triênio 1999-2001, 10\% dos municípios tinham CGMP inferior a 2,14. Já no triênio 2008-2010, o percentil $10 \%$ passa a ser de 3,5 . Da mesma forma, o percentil $10 \%$ da RZNV passa de 0,49 , no ano 2000, a 0,74, em 2009, com taxas anuais de variação de $5 \%$ e 4,2\%, respectivamente. Na Região Norte, os avanços são também nítidos: os limites críticos para o CGMP aumentam de 1,75 para 2,93 e, para a RZNV, de 0,38 para 0,64 , nos triênios inicial e final, com taxas de variação de $5,3 \%$ ao ano para ambos os indicadores.

Entre os estados do Nordeste, no triênio 19992001, o Maranhão apresentava a pior adequação dos dados de óbitos, em que $10 \%$ dos municípios tinham CGMP inferior a 0,9. Já no triênio 20082010 , o percentil $10 \%$ passa a ser de 2,7 . Igualmente, o percentil $10 \%$ da RZNV passa de 0,22 , em 2000, a 0,67, em 2009. Na Região Norte, todos os estados mostraram também acréscimos substanciais dos valores críticos inferiores tanto do CGMP quanto da RZNV.

Na Tabela 1, pode ser observada ainda a redução nos percentis $90 \%$ nos indicadores de regularidade (DMRO e DMRNV) para o conjunto das UF e regiões, do início para o final dos anos 2000. Nas regiões Norte e Nordeste, $10 \%$ dos municípios tinham valores de DMRO superiores a $40 \%$ no triênio 1999-2001, enquanto em 2008-2010 os percentis $90 \%$ decrescem para valores próximos a $20 \%$. A melhoria das informações do SINASC foi também expressiva, com valores críticos dos DMRNV decrescendo de valores próximos a $30 \%$ ao patamar de $13 \%$.

Mantendo o padrão de melhoria das informações vitais, avanços importantes relacionaram-se à redução na proporção de óbitos sem definição da causa básica em todas as UF. Enquanto no ano 2000, em 13 UF, 10\% dos municípios apresentavam proporção superior a $50 \%$ de óbitos mal definidos, em 2009, em apenas três estados, $10 \%$ dos municípios superavam $30 \%$. O 
Tabela 1

Percentil 10 do Coeficiente Geral de Mortalidade Padronizado por Idade (CGMP) e Razão de Nascidos Vivos Informados e Estimados (RZNV) e percentil 90 do Desvio Médio Relativo de Óbitos (DMRO), do Desvio Médio Relativo de Nascidos Vivos (DMRNV) e do percentual de óbitos mal definidos segundo Unidade da Federação (UF) e Região. Brasil, 1999-2001 e 2008-2010.

\begin{tabular}{|c|c|c|c|c|c|c|c|c|c|c|}
\hline \multirow[t]{3}{*}{ UF/Região } & \multicolumn{4}{|c|}{ Percentil 10} & \multicolumn{6}{|c|}{ Percentil 90} \\
\hline & \multicolumn{2}{|c|}{ CGMP } & \multicolumn{2}{|c|}{ RZNV } & \multicolumn{2}{|c|}{ DMRO } & \multicolumn{2}{|c|}{ DMRNV } & \multicolumn{2}{|c|}{$\begin{array}{l}\text { Percentual de óbitos } \\
\text { mal definidos }\end{array}$} \\
\hline & 1999-2001 & 2008-2010 & $1999-2001$ & 2008-2010 & 1999-2001 & $2008-2010$ & 1999-2001 & 2008-2010 & 1999-2001 & 2008-2010 \\
\hline $\mathrm{RO}$ & 1,9 & 3,4 & 0,52 & 0,64 & 44,6 & 34,3 & 23,0 & 15,0 & 27,2 & 13,8 \\
\hline$A C$ & 1,4 & 3,2 & 0,14 & 0,83 & 62,2 & 23,1 & 69,6 & 10,2 & 64,4 & 29,3 \\
\hline AM & 1,4 & 2,6 & 0,33 & 0,51 & 56,5 & 20,3 & 48,7 & 9,9 & 56,8 & 47,2 \\
\hline $\mathrm{RR}$ & 3,2 & 3,7 & 0,70 & 0,74 & 38,1 & 32,4 & 14,8 & 12,9 & 34,7 & 9,8 \\
\hline PA & 1,7 & 2,9 & 0,34 & 0,58 & 38,1 & 17,8 & 27,0 & 9,6 & 71,0 & 37,1 \\
\hline$A P$ & 0,9 & 1,7 & 0,27 & 0,54 & 80,9 & 25,6 & 44,2 & 22,1 & 51,5 & 26,4 \\
\hline TO & 2,6 & 3,3 & 0,55 & 0,71 & 46,4 & 31,4 & 24,7 & 16,7 & 56,0 & 12,0 \\
\hline Norte & 1,8 & 2,9 & 0,38 & 0,64 & 45,8 & 26,7 & 29,8 & 13,2 & 62,0 & 33,4 \\
\hline MA & 0,9 & 2,7 & 0,22 & 0,67 & 67,8 & 23,4 & 60,8 & 14,6 & 72,4 & 25,7 \\
\hline $\mathrm{PI}$ & 2,0 & 3,7 & 0,44 & 0,77 & 59,6 & 23,7 & 39,1 & 13,3 & 72,5 & 9,5 \\
\hline CE & 3,0 & 4,1 & 0,66 & 0,76 & 22,0 & 13,3 & 16,3 & 9,1 & 39,9 & 11,9 \\
\hline RN & 2,9 & 3,4 & 0,68 & 0,75 & 32,5 & 24,6 & 16,1 & 16,5 & 63,4 & 10,3 \\
\hline PB & 2,8 & 3,6 & 0,57 & 0,77 & 38,6 & 26,6 & 29,2 & 15,6 & 78,0 & 25,0 \\
\hline PE & 4,0 & 5,0 & 0,71 & 0,82 & 16,8 & 12,5 & 18,7 & 9,7 & 64,7 & 18,7 \\
\hline$A L$ & 3,7 & 4,1 & 0,67 & 0,74 & 24,8 & 18,8 & 14,8 & 10,7 & 64,1 & 19,2 \\
\hline SE & 3,5 & 4,9 & 0,70 & 0,81 & 33,0 & 16,0 & 17,6 & 11,3 & 60,8 & 13,6 \\
\hline BA & 2,3 & 3,3 & 0,52 & 0,72 & 27,7 & 18,6 & 25,8 & 12,0 & 67,3 & 37,0 \\
\hline Nordeste & 2,1 & 3,5 & 0,49 & 0,74 & 42,9 & 20,9 & 29,5 & 12,7 & 68,6 & 24,7 \\
\hline MG & 2,6 & 4,0 & 0,48 & 0,68 & 36,6 & 22,9 & 44,8 & 16,8 & 47,5 & 28,9 \\
\hline ES & 4,3 & 4,9 & 0,72 & 0,80 & 15,4 & 13,8 & 11,3 & 9,7 & 36,6 & 5,3 \\
\hline RJ & 5,6 & 5,8 & 0,80 & 0,74 & 11,6 & 12,5 & 10,7 & 9,1 & 15,7 & 11,3 \\
\hline SP & 4,6 & 4,9 & 0,76 & 0,77 & 19,9 & 17,1 & 14,2 & 13,5 & 22,3 & 20,8 \\
\hline Sudeste & 3,4 & 4,4 & 0,57 & 0,72 & 30,3 & 19,1 & 27,3 & 14,9 & 39,5 & 24,5 \\
\hline PR & 4,5 & 5,2 & 0,73 & 0,77 & 19,8 & 18,0 & 15,9 & 12,6 & 16,8 & 11,9 \\
\hline SC & 4,1 & 4,1 & 0,70 & 0,76 & 26,3 & 23,4 & 18,9 & 15,0 & 31,1 & 17,8 \\
\hline RS & 4,0 & 4,2 & 0,67 & 0,70 & 23,6 & 22,8 & 20,1 & 20,4 & 19,8 & 10,6 \\
\hline Sul & 4,1 & 4,3 & 0,71 & 0,74 & 22,2 & 21,5 & 18,2 & 16,3 & 23,2 & 12,8 \\
\hline MS & 4,3 & 4,8 & 0,57 & 0,73 & 21,0 & 15,7 & 16,8 & 13,2 & 18,7 & 5,0 \\
\hline MT & 3,4 & 3,1 & 0,54 & 0,63 & 33,3 & 22,2 & 20,8 & 16,6 & 12,8 & 11,1 \\
\hline GO & 3,1 & 3,9 & 0,57 & 0,63 & 32,6 & 27,1 & 25,7 & 20,0 & 27,3 & 15,9 \\
\hline DF & 5,5 & 5,2 & 1,05 & 1,02 & 1,0 & 2,0 & 1,8 & 0,3 & 4,9 & 1,5 \\
\hline $\begin{array}{l}\text { Centro- } \\
\text { oeste }\end{array}$ & 3,3 & 3,8 & 0,57 & 0,64 & 30,6 & 24,6 & 21,7 & 17,8 & 23,2 & 14,3 \\
\hline Brasil & 2,7 & 3,8 & 0,53 & 0,71 & 33,3 & 21,4 & 25,0 & 14,7 & 59,2 & 22,3 \\
\hline
\end{tabular}

UFs: AC - Acre; AL - Alagoas; AM - Amazonas; AP - Amapá; BA - Bahia; CE - Ceará; DF - Distrito Federal; ES - Espírito Santo; GO - Goiás; MA - Maranhão; MG - Minas Gerais; MS - Mato Grosso do Sul; MT - Mato Grosso; PA - Pará; PB - Paraíba; PE - Pernambuco; PI - Piauí; PR - Paraná; RJ - Rio de Janeiro; RN - Rio Grande do Norte; RO - Rondônia; RR - Roraima; RS - Rio Grande do Sul; SC - Santa Catarina; SE - Sergipe; SP - São Paulo; TO - Tocantins.

Amazonas mostrou a pior situação, com 10\% dos seus municípios com percentual de óbitos mal definidos superior a $47,2 \%$ (Tabela 1 ).
Nas Tabelas 2 e 3, estão apresentadas as proporções de municípios e da população residente por categoria de adequação das informações do SIM, segundo UF e regiões, para os anos de 
Tabela 2

Proporção (\%) de municípios por categoria de adequação das informações de mortalidade, segundo Unidade da Federação (UF) e Região. Brasil, 1999-2001 e 2008-2010.

\begin{tabular}{|c|c|c|c|c|c|c|}
\hline \multirow[t]{2}{*}{ UF/Região } & \multicolumn{2}{|c|}{ Categoria I * } & \multicolumn{2}{|c|}{ Categoria II ** } & \multicolumn{2}{|c|}{ Categoria III *** } \\
\hline & 1999-2001 & $2008-2010$ & 1999-2001 & 2008-2010 & 1999-2001 & 2008-2010 \\
\hline $\mathrm{RO}$ & 13,5 & 38,5 & 53,8 & 53,8 & 32,7 & 7,7 \\
\hline$A C$ & 0,0 & 27,3 & 45,5 & 72,7 & 54,5 & 0,0 \\
\hline AM & 1,6 & 8,1 & 45,2 & 64,5 & 53,2 & 27,4 \\
\hline $\mathrm{RR}$ & 20,0 & 13,3 & 73,3 & 86,7 & 6,7 & 0,0 \\
\hline PA & 2,1 & 25,2 & 54,5 & 62,9 & 43,4 & 11,9 \\
\hline $\mathrm{AP}$ & 12,5 & 6,3 & 0,0 & 62,5 & 87,5 & 31,2 \\
\hline TO & 2,9 & 28,1 & 77,0 & 66,9 & 20,1 & 5,0 \\
\hline Norte & 4,5 & 24,3 & 58,3 & 64,6 & 37,2 & 11,1 \\
\hline MA & 0,5 & 18,0 & 28,1 & 66,8 & 71,4 & 15,2 \\
\hline PI & 1,8 & 26,8 & 61,1 & 69,6 & 37,1 & 3,6 \\
\hline CE & 5,4 & 44,6 & 83,7 & 55,4 & 10,9 & 0,0 \\
\hline RN & 0,6 & 31,1 & 87,4 & 65,9 & 12,0 & 3,0 \\
\hline PB & 0,0 & 42,6 & 84,3 & 56,1 & 15,7 & 1,3 \\
\hline PE & 11,4 & 83,3 & 86,4 & 16,2 & 2,2 & 0,5 \\
\hline$A L$ & 4,0 & 61,7 & 92,0 & 37,3 & 4,0 & 1,0 \\
\hline SE & 4,0 & 82,7 & 93,3 & 17,3 & 2,7 & 0,0 \\
\hline BA & 4,6 & 29,5 & 72,5 & 65,2 & 22,9 & 5,3 \\
\hline Nordeste & 3,5 & 40,7 & 73,2 & 55,2 & 23,3 & 4,1 \\
\hline MG & 27,3 & 48,1 & 59,3 & 50,6 & 13,4 & 1,3 \\
\hline ES & 31,2 & 85,9 & 68,8 & 14,1 & 0,0 & 0,0 \\
\hline RJ & 93,4 & 98,9 & 6,6 & 1,1 & 0,0 & 0,0 \\
\hline SP & 67,6 & 76,4 & 32,1 & 23,6 & 0,3 & 0,0 \\
\hline Sudeste & 46,7 & 63,6 & 46,3 & 35,7 & 7,0 & 0,7 \\
\hline PR & 65,4 & 82,7 & 34,1 & 17,3 & 0,5 & 0,0 \\
\hline SC & 38,6 & 53,6 & 60,4 & 46,4 & 1,0 & 0,0 \\
\hline RS & 46,1 & 55,8 & 52,2 & 43,6 & 1,7 & 0,6 \\
\hline Sul & 50,8 & 64,3 & 48,1 & 35,4 & 1,1 & 0,3 \\
\hline MS & 54,5 & 80,8 & 45,5 & 19,2 & 0,0 & 0,0 \\
\hline $\mathrm{MT}$ & 36,5 & 51,1 & 58,7 & 40,4 & 4,8 & 8,5 \\
\hline GO & 25,2 & 49,2 & 67,4 & 49,2 & 7,4 & 1,6 \\
\hline DF & 100,0 & 100,0 & 0,0 & 0,0 & 0,0 & 0,0 \\
\hline Centro-oeste & 33,6 & 55,2 & 61,0 & 41,4 & 5,4 & 3,4 \\
\hline Brasil & 29,1 & 52,5 & 57,5 & 44,8 & 13,4 & 2,7 \\
\hline
\end{tabular}

UFs: AC - Acre; AL - Alagoas; AM - Amazonas; AP - Amapá; BA - Bahia; CE - Ceará; DF - Distrito Federal; ES - Espírito Santo; GO - Goiás; MA - Maranhão; MG - Minas Gerais; MS - Mato Grosso do Sul; MT - Mato Grosso; PA - Pará;

PB - Paraíba; PE - Pernambuco; PI - Piauí; PR - Paraná; RJ - Rio de Janeiro; RN - Rio Grande do Norte; RO - Rondônia;

RR - Roraima; RS - Rio Grande do Sul; SC - Santa Catarina; SE - Sergipe; SP - São Paulo; TO - Tocantins.

* Informações consideradas adequadas;

** Informações não adequadas;

*** Informações deficientes.

2000 e 2009, respectivamente. Verifica-se que, de maneira geral, há um incremento importante de municípios e população residente na Categoria I (informações vitais consideradas adequadas), e um descenso expressivo na Categoria III (informações vitais deficientes). Alguns estados que dispõem de número reduzido de municípios como Roraima e Amapá constituíram exceções, com decréscimos na proporção de municípios e na população residente na Categoria I. No triênio 1999-2001, esses estados, que concentram proporção expressiva de população residente, apre- 
Tabela 3

Proporção (\%) da população residente por categoria de adequação das informações de mortalidade e Índice de Adequação, segundo Unidade da Federação (UF) e Região. Brasil, 1999-2001 e 2008-2010.

\begin{tabular}{|c|c|c|c|c|c|c|c|c|}
\hline \multirow[t]{2}{*}{ UF/Região } & \multicolumn{2}{|c|}{ Categoria I * } & \multicolumn{2}{|c|}{ Categoria II ** } & \multicolumn{2}{|c|}{ Categoria III *** } & \multicolumn{2}{|c|}{ Índice de adequação } \\
\hline & 1999-2001 & 2008-2010 & 1999-2001 & 2008-2010 & 1999-2001 & 2008-2010 & 1999-2001 & 2008-2010 \\
\hline $\mathrm{RO}$ & 41,5 & 71,0 & 48,4 & 26,8 & 10,1 & 2,2 & 55,6 & 82,2 \\
\hline$A C$ & 0,0 & 67,5 & 84,4 & 32,5 & 15,6 & 0,0 & 26,6 & 83,8 \\
\hline AM & 50,0 & 55,8 & 25,4 & 34,2 & 24,6 & 10,0 & 38,1 & 62,9 \\
\hline RR & 69,7 & 64,8 & 28,5 & 35,2 & 1,8 & 0,0 & 82,2 & 82,4 \\
\hline PA & 22,1 & 54,1 & 53,0 & 39,2 & 24,9 & 6,7 & 23,7 & 67,0 \\
\hline$A P$ & 76,3 & 58,5 & 0,0 & 34,8 & 23,7 & 6,7 & 52,6 & 69,2 \\
\hline TO & 26,2 & 64,8 & 62,7 & 33,3 & 11,1 & 1,9 & 46,5 & 79,6 \\
\hline Norte & 32,9 & 58,1 & 46,1 & 35,8 & 21,0 & 6,1 & 35,0 & 69,9 \\
\hline MA & 15,4 & 47,0 & 41,1 & 44,7 & 43,5 & 8,3 & $-7,6$ & 61,1 \\
\hline $\mathrm{PI}$ & 25,8 & 56,4 & 52,4 & 42,4 & 21,8 & 1,2 & 30,2 & 76,4 \\
\hline CE & 32,3 & 67,1 & 62,2 & 32,9 & 5,5 & 0,0 & 57,9 & 83,6 \\
\hline RN & 25,7 & 67,4 & 68,0 & 31,7 & 6,3 & 0,9 & 53,4 & 82,4 \\
\hline PB & 0,0 & 74,3 & 94,7 & 25,3 & 5,3 & 0,4 & 42,1 & 86,6 \\
\hline PE & 46,5 & 93,1 & 52,8 & 6,9 & 0,7 & 0,0 & 72,2 & 96,6 \\
\hline $\mathrm{AL}$ & 30,8 & 78,6 & 67,2 & 21,2 & 2,0 & 0,2 & 62,4 & 89,0 \\
\hline SE & 33,4 & 94,9 & 66,0 & 5,1 & 0,6 & 0,0 & 65,8 & 97,5 \\
\hline BA & 29,7 & 55,7 & 57,3 & 41,9 & 13,0 & 2,4 & 45,4 & 74,3 \\
\hline Nordeste & 28,8 & 67,5 & 59,3 & 30,7 & 11,9 & 1,8 & 46,6 & 81,1 \\
\hline MG & 63,3 & 75,8 & 32,1 & 23,8 & 4,6 & 0,4 & 74,8 & 87,3 \\
\hline ES & 66,7 & 96,1 & 33,3 & 3,9 & 0,0 & 0,0 & 83,4 & 98,1 \\
\hline RJ & 99,3 & 99,8 & 0,7 & 0,2 & 0,0 & 0,0 & 99,7 & 99,9 \\
\hline SP & 94,2 & 95,1 & 5,8 & 4,9 & 0,0 & 0,0 & 97,1 & 97,6 \\
\hline Sudeste & 86,5 & 91,3 & 12,4 & 8,6 & 1,1 & 0,1 & 91,6 & 95,5 \\
\hline PR & 86,1 & 96,6 & 13,8 & 3,4 & 0,1 & 0,0 & 92,9 & 98,3 \\
\hline SC & 57,6 & 77,3 & 42,3 & 22,7 & 0,1 & 0,0 & 78,7 & 88,7 \\
\hline RS & 85,1 & 89,7 & 14,6 & 10,2 & 0,3 & 0,1 & 92,1 & 94,7 \\
\hline Sul & 79,6 & 89,7 & 20,2 & 10,3 & 0,2 & 0,0 & 89,5 & 94,9 \\
\hline MS & 79,4 & 94,8 & 20,6 & 5,2 & 0,0 & 0,0 & 89,7 & 97,4 \\
\hline MT & 70,1 & 79,2 & 28,6 & 18,8 & 1,3 & 2,0 & 83,1 & 86,6 \\
\hline GO & 64,9 & 85,7 & 33,9 & 14,1 & 1,2 & 0,2 & 80,7 & 92,6 \\
\hline DF & 100,0 & 100,0 & 0,0 & 0,0 & 0,0 & 0,0 & 100,0 & 100,0 \\
\hline Centro-oeste & 74,8 & 88,5 & 24,4 & 11,0 & 0,8 & 0,5 & 86,2 & 93,5 \\
\hline Brasil & 64,3 & 81,5 & 30,2 & 17,4 & 5,5 & 1,1 & 73,9 & 89,1 \\
\hline
\end{tabular}

UFs: AC - Acre; AL - Alagoas; AM - Amazonas; AP - Amapá; BA - Bahia; CE - Ceará; DF - Distrito Federal; ES - Espírito Santo; GO - Goiás; MA - Maranhão; MG - Minas Gerais; MS - Mato Grosso do Sul; MT - Mato Grosso; PA - Pará; PB - Paraíba; PE - Pernambuco; PI - Piauí; PR - Paraná; RJ - Rio de Janeiro; RN - Rio Grande do Norte; RO - Rondônia; RR - Roraima; RS - Rio Grande do Sul; SC - Santa Catarina; SE - Sergipe; SP - São Paulo; TO - Tocantins.

* Informações consideradas adequadas;

** Informações não adequadas;

*** Informações deficientes.

sentavam indicadores melhores do que a média da Região Norte. Já no triênio 2008-2010, com a melhora de todos os indicadores na região, os indicadores dos dois estados se estabeleceram no mesmo patamar.
Chamam a atenção os avanços na Região Nordeste, sobretudo nos estados de Sergipe, Pernambuco e Alagoas, onde a maior parte da população residente passou a ser classificada na Categoria I (Tabelas 2 e 3). 
Apesar das melhorias encontradas de aumento na Categoria I e redução na Categoria III, as proporções na categoria intermediária (informações vitais não adequadas) permanecem elevadas no geral (Tabelas 2 e 3 ).

A análise do índice de adequação das informações sobre mortalidade mostrou aumentos em todas as UF e regiões do país, havendo ampliação mais substancial naquelas que, no ano 2000, apresentavam pior nível de adequação. Enquanto no ano de referência inicial (2000) apenas dez UF tinham índices superiores a $80 \%$, em 2009 o número aumentou para 20 UF (Tabela 3). O menor valor do índice de adequação, em 2009, foi de 61,1, para o Estado do Maranhão, que apresentava índice negativo em 2000. No ano de 2009, Sergipe, Pernambuco, Espírito Santo, Rio de Janeiro, São Paulo, Paraná, Rio Grande do Sul, Mato Grosso do Sul, Goiás e Distrito Federal apresentaram índices superiores a $90 \%$.

No Brasil, o índice de adequação das informações de óbitos passou de 73,9 para 89,1, com taxa de variação anual de 1,9\%. Nas regiões Norte e Nordeste, as taxas anuais de variação do índice de adequação foram, respectivamente, $7,2 \%$ e $5,7 \%$, enquanto, nas demais regiões, as taxas ficaram abaixo de $1 \%$ ao ano.

As proporções de municípios na Categoria I das informações do SINASC aumentaram na maioria das UF e em todas as regiões, enquanto as proporções na Categoria III diminuíram em todas as UF entre 2000 e 2009. No Maranhão, a proporção de municípios com informações adequadas de nascidos vivos (Categoria I) passou de $8,8 \%$ para $61,7 \%$, e a proporção de municípios com informações deficientes decresce de $60,4 \%$ para 2,8\%. É importante destacar o fato de que, em 2009, 22 UF têm proporções abaixo de 5\% na Categoria III (Tabela 4).

A análise referente à proporção da população residente por categoria de adequação das informações sobre nascidos vivos mostrou, de igual maneira, progressos relevantes. Nas regiões Norte e Nordeste, as proporções de população residente em municípios com informações adequadas crescem de cerca de $50 \%$ para o patamar de $80 \%$, no período de 10 anos. Quanto ao índice de adequação das informações de nascidos vivos, encontrou-se uma melhoria em praticamente todas as UF. Em 2009, o valor mínimo do índice de adequação para nascidos vivos foi de 79,1, para o Pará, e 15 UF apresentaram índices superiores a 90 (Tabela 5).

No Brasil, o índice de adequação cresceu de 80,5 para 90,9, com taxa de variação anual de $1,2 \%$. Nas regiões Norte e Nordeste, as taxas anuais de variação do índice de adequação foram, respectivamente, $5,2 \%$ e $3,1 \%$, enquanto nas demais regiões, as taxas ficaram abaixo de $0,5 \%$ ao ano.

\section{Discussão}

A análise do nível de adequação das informações vitais no Brasil demonstrou inequívoca melhoria da situação em todo o país, na primeira década deste século, com particular incremento nas regiões com pior situação, as regiões Norte e Nordeste e suas respectivas UF.

Os resultados do presente estudo indicam que grande parte dos municípios e da população total reside, atualmente, em UF que dispõem de informações adequadas, tanto de nascimentos quanto de óbitos. Os indicadores traçadores da cobertura e regularidade das informações apontam o quanto se avançou a partir do ano 2000, no conjunto das UF e regiões, confirmando resultados de avaliação prévia 11. Estudos sobre os sistemas de informações vitais já sinalizavam melhoria para diferentes recortes geográficos, utilizando diversas abordagens histórico-descritivas e metodológicas 19,25,26,27,28.

Da mesma forma, os resultados aqui encontrados corroboram os aumentos nas coberturas do SIM e SINASC, identificados nos procedimentos de correção das estatísticas vitais com base na busca ativa de óbitos e nascimentos na Amazônia Legal e Nordeste ${ }^{8}$. Tal como as coberturas, os valores dos índices de adequação estão próximos a 90\%, tanto para as informações de óbitos quanto de nascidos vivos; contudo a homogeneidade dos índices de nascidos vivos é bem maior. Em 2009, apenas uma UF apresentou índice de adequação das informações de nascidos vivos menor que 80, enquanto sete UF apresentaram índices de adequação de informações de mortalidade inferiores a 80.

Percebe-se, assim, que os indicadores construídos com dados dos próprios sistemas foram úteis para avaliar a adequação das informações. Esses indicadores são fáceis de calcular e servem para o estabelecimento de metas a serem alcançadas 29. Por outro lado, prestam-se a sinalizar pontos críticos por UF, que podem subsidiar oportunidades de intervenção com vistas à superação dos problemas relacionados à cobertura incompleta, à irregularidade das notificações dos eventos e à baixa qualidade dos dados sobre causa de morte. No entanto, é preciso ter em conta que os indicadores utilizados são mais sujeitos a flutuações nos estados que dispõem de número reduzido de municípios, a exemplo de Roraima e Amapá. Portanto, os resultados devem ser examinados à luz dessa limitação. 
Tabela 4

Proporção (\%) de municípios por categoria de adequação das informações de nascidos vivos, segundo Unidade da Federação (UF) e Região. Brasil, 1999-2001 e 2008-2010.

\begin{tabular}{|c|c|c|c|c|c|c|}
\hline \multirow[t]{2}{*}{ UF/Região } & \multicolumn{2}{|c|}{ Categoria I * } & \multicolumn{2}{|c|}{ Categoria II ** } & \multicolumn{2}{|c|}{ Categoria III *** } \\
\hline & 1999-2001 & 2008-2010 & 1999-2001 & 2008-2010 & 1999-2001 & 2008-2010 \\
\hline $\mathrm{RO}$ & 34,6 & 46,2 & 50,0 & 50,0 & 15,4 & 3,8 \\
\hline$A C$ & 18,1 & 90,9 & 36,4 & 9,1 & 45,5 & 0,0 \\
\hline AM & 8,1 & 38,7 & 32,3 & 48,4 & 59,6 & 12,9 \\
\hline RR & 66,7 & 80,0 & 33,3 & 20,0 & 0,0 & 0,0 \\
\hline PA & 18,9 & 53,1 & 33,6 & 36,4 & 47,5 & 10,5 \\
\hline AP & 18,8 & 37,5 & 43,8 & 50,0 & 37,4 & 12,5 \\
\hline TO & 35,3 & 54,7 & 50,3 & 43,9 & 14,4 & 1,4 \\
\hline Norte & 25,8 & 53,0 & 41,0 & 40,5 & 33,2 & 6,5 \\
\hline MA & 8,8 & 61,7 & 30,8 & 35,5 & 60,4 & 2,8 \\
\hline $\mathrm{PI}$ & 24,4 & 69,6 & 46,6 & 29,5 & 29,0 & 0,9 \\
\hline CE & 46,7 & 65,2 & 48,4 & 32,1 & 4,9 & 2,7 \\
\hline RN & 44,6 & 60,5 & 50,6 & 38,9 & 4,8 & 0,6 \\
\hline PB & 24,7 & 70,4 & 62,7 & 28,7 & 12,6 & 0,9 \\
\hline PE & 61,1 & 86,5 & 34,6 & 13,0 & 4,3 & 0,5 \\
\hline $\mathrm{AL}$ & 48,5 & 65,7 & 48,5 & 33,3 & 3,0 & 1,0 \\
\hline SE & 56,0 & 74,7 & 40,0 & 25,3 & 4,0 & 0,0 \\
\hline BA & 28,4 & 58,3 & 51,4 & 39,5 & 20,2 & 2,2 \\
\hline Nordeste & 34,1 & 66,6 & 47,0 & 31,9 & 18,9 & 1,5 \\
\hline MG & 32,7 & 51,0 & 45,5 & 45,6 & 21,8 & 3,4 \\
\hline ES & 68,8 & 80,7 & 28,6 & 16,7 & 2,6 & 2,6 \\
\hline RJ & 78,0 & 68,5 & 22,0 & 31,5 & 0,0 & 0,0 \\
\hline SP & 71,8 & 70,3 & 27,6 & 28,8 & 0,6 & 0,9 \\
\hline Sudeste & 52,0 & 60,8 & 36,5 & 37,0 & 11,5 & 2,2 \\
\hline PR & 61,7 & 68,4 & 36,8 & 31,6 & 1,5 & 0,0 \\
\hline SC & 57,0 & 63,8 & 39,6 & 36,2 & 3,4 & 0,0 \\
\hline RS & 54,6 & 49,2 & 40,7 & 48,4 & 4,7 & 2,4 \\
\hline Sul & 57,6 & 59,3 & 39,1 & 39,7 & 3,3 & 1,0 \\
\hline MS & 51,9 & 66,6 & 35,1 & 30,8 & 13,0 & 2,6 \\
\hline MT & 42,1 & 41,8 & 44,4 & 50,4 & 13,5 & 7,8 \\
\hline GO & 47,9 & 43,9 & 39,3 & 48,8 & 12,8 & 7,3 \\
\hline DF & 100,0 & 100,0 & 0,0 & 0,0 & 0,0 & 0,0 \\
\hline Centro-oeste & 47,1 & 47,2 & 39,9 & 46,1 & 13,0 & 6,7 \\
\hline Brasil & 44,8 & 60,6 & 41,1 & 37,0 & 14,1 & 2,4 \\
\hline
\end{tabular}

UFs: AC - Acre; AL - Alagoas; AM - Amazonas; AP - Amapá; BA - Bahia; CE - Ceará; DF - Distrito Federal; ES - Espírito Santo; GO - Goiás; MA - Maranhão; MG - Minas Gerais; MS - Mato Grosso do Sul; MT - Mato Grosso; PA - Pará; PB - Paraíba; PE - Pernambuco; PI - Piauí; PR - Paraná; RJ - Rio de Janeiro; RN - Rio Grande do Norte; RO - Rondônia; RR - Roraima; RS - Rio Grande do Sul; SC - Santa Catarina; SE - Sergipe; SP - São Paulo; TO - Tocantins.

* Informações consideradas adequadas;

** Informações não adequadas;

*** Informações deficientes.

Com relação às causas de morte mal definidas, foram achados substanciais progressos, especialmente no Norte e Nordeste 6 . A ampliação do acesso às políticas públicas inclusivas, em particular ao sistema de saúde, de expressiva parcela da população brasileira contribuiu para a melhoria da definição da causa básica. Entre as ações assistenciais que ajudaram o avanço na qualidade das informações se destacam a institucionalização dos partos em maternidades, na 
Tabela 5

Proporção (\%) da população total de residentes por categoria de adequação das informações de nascidos vivos e Índice de Adequação, segundo Unidade da Federação (UF) e Região. Brasil, 1999-2001 e 2008-2010.

\begin{tabular}{|c|c|c|c|c|c|c|c|c|}
\hline \multirow[t]{2}{*}{ UF/Região } & \multicolumn{2}{|c|}{ Categoria I * } & \multicolumn{2}{|c|}{ Categoria II ** } & \multicolumn{2}{|c|}{ 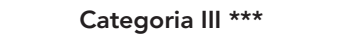 } & \multicolumn{2}{|c|}{ Índice de adequação } \\
\hline & 1999-2001 & 2008-2010 & 1999-2001 & 2008-2010 & 1999-2001 & 2008-2010 & 1999-2001 & 2008-2010 \\
\hline $\mathrm{RO}$ & 71,1 & 76,8 & 22,1 & 21,4 & 6,8 & 1,8 & 75,4 & 85,7 \\
\hline$A C$ & 61,6 & 95,1 & 21,4 & 4,9 & 17,0 & 0,0 & 55,3 & 97,6 \\
\hline AM & 54,5 & 74,0 & 21,5 & 20,8 & 24,0 & 5,2 & 41,3 & 79,2 \\
\hline RR & 86,1 & 86,6 & 13,9 & 13,4 & 0,0 & 0,0 & 93,1 & 93,3 \\
\hline PA & 49,6 & 73,4 & 25,6 & 21,5 & 24,8 & 5,1 & 37,6 & 79,1 \\
\hline AP & 77,0 & 85,2 & 16,2 & 12,9 & 6,8 & 1,9 & 78,3 & 89,8 \\
\hline TO & 69,1 & 77,9 & 25,3 & 21,1 & 5,6 & 1,0 & 76,2 & 87,5 \\
\hline Norte & 57,2 & 76,1 & 23,5 & 20,0 & 19,3 & 3,9 & 49,7 & 82,2 \\
\hline MA & 25,2 & 79,7 & 39,1 & 18,9 & 35,7 & 1,4 & 9,1 & 87,8 \\
\hline PI & 60,7 & 86,1 & 26,7 & 13,5 & 12,6 & 0,4 & 61,5 & 92,5 \\
\hline CE & 66,8 & 78,7 & 31,1 & 19,8 & 2,1 & 1,5 & 80,3 & 87,1 \\
\hline RN & 70,6 & 80,4 & 27,0 & 19,5 & 2,4 & 0,1 & 81,7 & 90,1 \\
\hline PB & 40,1 & 82,2 & 53,8 & 17,4 & 6,1 & 0,4 & 60,9 & 90,5 \\
\hline PE & 78,6 & 84,0 & 20,1 & 16,0 & 1,3 & 0,0 & 87,4 & 92,0 \\
\hline $\mathrm{AL}$ & 71,7 & 81,7 & 27,4 & 18,0 & 0,9 & 0,3 & 84,5 & 90,4 \\
\hline SE & 79,7 & 90,1 & 18,7 & 9,9 & 1,6 & 0,0 & 87,5 & 95,1 \\
\hline BA & 53,2 & 76,3 & 36,2 & 23,0 & 10,6 & 0,7 & 60,7 & 87,1 \\
\hline Nordeste & 58,8 & 80,4 & 32,1 & 18,9 & 9,1 & 0,7 & 65,8 & 89,2 \\
\hline MG & 70,6 & 76,2 & 22,5 & 22,8 & 6,9 & 1,0 & 75,0 & 86,6 \\
\hline ES & 88,2 & 94,2 & 11,3 & 5,0 & 0,5 & 0,8 & 93,4 & 95,9 \\
\hline RJ & 89,9 & 95,1 & 10,1 & 4,9 & 0,0 & 0,0 & 95,0 & 97,6 \\
\hline SP & 95,8 & 94,7 & 4,1 & 5,2 & 0,1 & 0,1 & 97,8 & 97,2 \\
\hline Sudeste & 88,1 & 90,2 & 10,1 & 9,5 & 1,8 & 0,3 & 91,4 & 94,7 \\
\hline PR & 82,3 & 80,2 & 17,3 & 19,8 & 0,4 & 0,0 & 90,6 & 90,1 \\
\hline SC & 81,3 & 87,8 & 17,4 & 12,2 & 1,3 & 0,0 & 88,7 & 93,9 \\
\hline RS & 83,1 & 80,2 & 16,1 & 19,4 & 0,8 & 0,4 & 90,4 & 89,5 \\
\hline Sul & 82,5 & 81,9 & 16,8 & 18,0 & 0,7 & 0,1 & 90,2 & 90,8 \\
\hline MS & 81,7 & 86,5 & 14,4 & 12,5 & 3,9 & 1,0 & 85,0 & 91,8 \\
\hline MT & 72,3 & 73,5 & 23,1 & 24,8 & 4,6 & 1,7 & 79,3 & 84,2 \\
\hline GO & 75,6 & 67,2 & 21,9 & 31,5 & 2,5 & 1,3 & 84,1 & 81,7 \\
\hline DF & 100,0 & 100,0 & 0,0 & 0,0 & 0,0 & 0,0 & 100,0 & 100,0 \\
\hline Centro-oeste & 80,3 & 78,0 & 16,9 & 20,9 & 2,8 & 1,1 & 86,0 & 87,4 \\
\hline Brasil & 76,2 & 84,2 & 18,7 & 15,0 & 5,1 & 0,8 & 80,5 & 90,9 \\
\hline
\end{tabular}

UFs: AC - Acre; AL - Alagoas; AM - Amazonas; AP - Amapá; BA - Bahia; CE - Ceará; DF - Distrito Federal; ES - Espírito Santo; GO - Goiás; MA - Maranhão; MG - Minas Gerais; MS - Mato Grosso do Sul; MT - Mato Grosso; PA - Pará; PB - Paraíba; PE - Pernambuco; PI - Piauí; PR - Paraná; RJ - Rio de Janeiro; RN - Rio Grande do Norte; RO - Rondônia; RR - Roraima; RS - Rio Grande do Sul; SC - Santa Catarina; SE - Sergipe; SP - São Paulo; TO - Tocantins.

* Informações consideradas adequadas;

** Informações não adequadas:

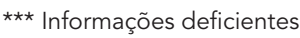

quase totalidade dos municípios, maior acesso à rede de urgência, emergência e hospitalar, além do programa de saúde da família, em especial nas regiões Norte e Nordeste, que aproxima grupos desfavorecidos dos serviços de saúde 17,30.
Os avanços alcançados nos anos 2000 expressam, sem dúvida, o consenso construído quanto à relevância de se dispor de informações válidas, confiáveis e oportunas para todo o país, considerando diferentes níveis de desagregação geográ- 
fica 4 . Aliados às recomendações de organismos internacionais para que se proceda à revisão e consolidação dos sistemas de informação, de forma a permitir a avaliação da cobertura e da qualidade das informações, os dados vitais brasileiros encontram-se, hoje, em nível satisfatório para a construção de indicadores de desenvolvimento e para avaliar políticas de saúde, salvo poucas exceções. De forma semelhante, outros países vêm avançando com seus sistemas de informação 31,32, embora várias nações ainda permaneçam dependentes de inquéritos populacionais para conhecer a magnitude de sua mortalidade 10 .

Os achados do presente trabalho mostram que as estratégias adotadas pelo Brasil para fortalecer os sistemas de informações vitais de registro contínuo do Ministério da Saúde se mostraram efetivas. Ações nos níveis federal, estadual e municipal, com gestão partilhada e grande apoio acadêmico e da Rede Interagencial de Informações para a Saúde (RIPSA) foram imprescindíveis para seu êxito. Pelo reconhecimento do valor das avaliações dos dados por município os investimentos foram direcionados, prioritariamente, para localidades com maior precariedade das informações 4,33,34. Além disso, a incorporação da busca ativa de eventos na rotina dos municípios, o aperfeiçoamento da vigilância do óbito, da autópsia verbal em localidades desassistidas, a integração com os comitês de prevenção do óbito materno, fetal e infantil e a capacitação dos envolvidos na produção da informação são exemplos de intervenções estratégicas que contribuem para reduzir a desigualdade nos níveis de adequação das informações vitais 21,35,36.

Com a análise de adequação das informações vitais utilizando indicadores dos próprios sistemas, espera-se induzir a um círculo virtuoso: ao valorizar a produção dos dados gerados pelos serviços, contribui-se para o seu aperfeiçoamento. Ao identificar os municípios com informação precária, expõem-se as iniquidades, instigando o desenvolvimento de intervenções para sua superação.

Apesar dos avanços obtidos na primeira década do século XXI, persistem desafios para o alcance de cobertura universal em todos os municípios brasileiros. No que diz respeito à qualidade, novos desafios se descortinam, como, por exemplo, a melhoria no nível de detalhamento da classificação da causa básica de morte. Além de ações direcionadas à melhoria dos registros vitais, alcançar o nível ótimo continuará na dependência do poder público e da sociedade em garantir acesso equânime às políticas públicas a toda população.

\section{Resumen}

Los sistemas de información sobre mortalidad (SIM) y nacidos vivos (SINASC) del Ministerio de Salud constituyen instrumentos de medición de la situación de salud. El objetivo de este artículo es evaluar el nivel de adecuación de los sistemas de información vitales entre los trienios 1999-2001 y 2008-2010 en las Unidades de la Federación (UF) y regiones brasileñas. En base a los datos del SIM y SINASC, se construyeron indicadores para evaluar la cobertura, la regularidad y la calidad de la información. Los municipios fueron clasificados en tres categorías, de acuerdo con la adecuación de óbitos y nacidos vivos, calculándose un índice de adecuación basado en la proporción de la población residente en cada una de las categorías. El Índice de Adecuación para Brasil aumentó de 73,9 a 89,1 (SIM) y de 80,5 a 90, 9 (SINASC), entre los dos trienios, respectivamente. Los avances se produjeron en todos los indicadores, en todas las regiones y gran parte de las UF. El análisis del nivel de adecuación de la información vital en Brasil demostró una inequívoca mejoría de la situación en todo el país, particularmente en las UF de los regiones Norte y Nordeste.

Sistemas de Información; Estadísticas Vitales, Evaluación en Salud; Nacimiento Vivo 


\section{Colaboradores}

P. G. Frias contribuiu na concepção do artigo, pesquisa bibliográfica, análise dos dados, redação do artigo e aprovou o texto final. C. L. Szwarcwald contribuiu na concepção do artigo, pesquisa bibliográfica, análise dos dados, redação e aprovou o texto final. P. I. C. Lira participou da concepção do artigo, análise, revisão crítica do conteúdo e aprovou o texto final.

\section{Agradecimentos}

Ao Ministério da Saúde pela disponibilização dos dados no site do DATASUS que possibilitaram a análise.

\section{Referências}

1. Barros FC, Matijasevich A, Requejo JH, Giugliani E, Maranhão AG, Monteiro CA, et al. Recent trends in maternal, newborn, and child health in Brazil: progress toward Millennium Development Goals 4 and 5. Am J Public Health 2010; 100:1877-89.

2. Murray CJ, Rajaratnam JK, Marcus J, Laakso T, Lopez AD. What can we conclude from death registration? Improved methods for evaluating completeness. PLoS Med 2010; 7:e1000262.

3. Mathers C, Boerma T. Mortality measurement matters: improving data collection and estimation methods for child and adult mortality. PLoS Med 2010; 7:e1000265.

4. Frias PG, Szwarcwald CL, Lira PIC. Estimação da mortalidade infantil no contexto de descentralização do Sistema Único de Saúde (SUS). Rev Bras Saúde Matern Infant 2011; 11:463-70.

5. Schmidt MI, Duncan BB, Silva GA, Menezes AM, Monteiro CA, Barreto SM, et al. Chronic non-communicable diseases in Brazil: burden and current challenges. Lancet 2011; 377:1949-61.
6. França E, de Abreu DX, Rao C, Lopez AD. Evaluation of cause-of-death statistics for Brazil, 20022004. Int J Epidemiol 2008; 37:891-901.

7. Alkema L, You D. Child mortality estimation: a comparison of UN IGME and IHME estimates of levels and trends in under-five mortality rates and deaths. PLoS Med 2012; 9:e1001288.

8. Frias PG, Szwarcwald CL, Souza Jr. PRB, Almeida WS, Lira PIC. Correção das informações vitais no Brasil: estimação da mortalidade infantil, 20002009. Rev Saúde Pública 2013; 47:1048-58.

9. Hill AG, David PH. Monitoring changes in child mortality: new methods for use in developing countries. Health Policy Plan 1988; 3:214-26.

10. Silva R. Child mortality estimation: consistency of under-five mortalitiy rate estimates using full birth histories and summary birth histories. PLoS Med 2012; 9:e1001296.

11. Szwarcwald CL. Strategies for improving the monitoring of vital events in Brazil. Int J Epidemiol 2008; 37:738-44. 
12. Murray CJ, Laakso T, Shibuya K, Hill K, Lopez AD. Can we achieve Millennium Development Goal 4 ? New analysis of country trends and forecasts of under-5 mortality to 2015 . Lancet $2007 ; 370$ : 1040-54.

13. Hill K, You D, Inoue M, Oestergaard MZ; Technical Advisory Group of United Nations Inter-agency Group for Child Mortality Estimation. Child mortality estimation: accelerated progress in reducing global child mortality, 1990-2010. PLoS Med 2012; 9:e1001303.

14. Frias PG, Pereira PMH, Andrade CLT, Lira PIC, Szwarcwald CL. Avaliação da adequação das informações de mortalidade e nascidos vivos no Estado de Pernambuco, Brasil. Cad Saúde Pública 2010; 26:671-81.

15. Szwarcwald CL, Leal MC, Andrade CLT, Souza Jr. PRB. Estimação da mortalidade infantil no Brasil: o que dizem as informações sobre óbitos e nascimentos do Ministério da Saúde? Cad Saúde Pública 2002 ; 18:1725-36.

16. Mello-Jorge MHP, Laurenti R, Gotlieb SLD. Análise da qualidade das estatísticas vitais brasileiras: a experiência de implantação do SIM e do SINASC. Ciênc Saúde Coletiva 2007; 12:643-54.

17. Victora CG, Aquino EM, Leal MC, Monteiro CA, Barros FC, Szwarcwald CL. Maternal and child health in Brazil: progress and challenges. Lancet 2011; 377:1863-76.

18. Secretaria de Vigilância à Saúde, Ministério da Saúde. Manual para investigação do óbito com causa mal definida. Brasília: Ministério da Saúde; 2009.

19. Lima CRA, Schramm JMA, Coeli CM, Silva MEM. Revisão das dimensões de qualidade dos dados e métodos aplicados na avaliação dos sistemas de informação em saúde. Cad Saúde Pública 2009; 25:2095-109.

20. Frias PG, Vidal AS, Pereira PMH, Lira PIC, Vanderlei LC. Avaliação da notificação de óbitos infantis ao Sistema de Informação sobre Mortalidade: um estudo de caso. Rev Bras Saúde Matern Infant 2005; 5:43-51.

21. Campos D, França E, Loschi RH, Souza MFM. Uso da autópsia verbal na investigação de óbitos com causa mal definida em Minas Gerais, Brasil. Cad Saúde Pública 2010; 26:1221-33.

22. Szwarcwald CL, Morais Neto OL, Frias PG, Souza Jr. PRB, Escalante JJC, Lima RB, et al. Busca ativa de óbitos e nascimentos no Nordeste e na Amazônia Legal: estimação das coberturas do SIM e do SINASC nos municípios brasileiros. In: Ministério da Saúde, organizador. Saúde Brasil 2011: uma análise da situação de saúde. Brasília: Ministério da Saúde; 2011. p. 79-97.
23. Andrade CLT, Szwarcwald CL. Desigualdades socioespaciais da adequação das informações de nascimentos e óbitos do Ministério da Saúde, Brasil, 2000-2002. Cad Saúde Pública 2007; 23: 1207-16.

25. United Nations. Model life tables for developing countries. http://www.un.org/esa/population/ techcoop/DemMod/model_lifetabs/model_life tabs.html (acessado em 22/Nov/2013).

26. Mello-Jorge MHP, Laurenti R, Gotlieb SLD. Avaliação dos sistemas de informações em saúde no Brasil. Cad Saúde Colet (Rio J) 2010; 18:7-18.

27. Paes N. Avaliação da cobertura dos registros de óbitos dos estados brasileiros em 2000. Rev Saúde Pública 2005; 39:882-90.

28. Pedraza DF. Qualidade do Sistema de Informações sobre Nascidos Vivos (SINASC): análise crítica da literatura. Ciênc Saúde Coletiva 2012; 17:2729-37.

29. Figueiroa BQ, Vanderlei LCM, Frias PG, Carvalho PI, Szwarcwald CL. Análise da cobertura do Sistema de Informações sobre Mortalidade em Olinda, Pernambuco, Brasil. Cad Saúde Pública 2013; 29:475-84.

30. Rodrigues M, Bonfim C, Frias PG, Braga C, Gurgel IGD, Medeiros Z. Differentials in vital information in the state of Pernambuco, Brazil, 2006-2008. Rev Bras Epidemiol 2012; 15:275-84.

31. Victora CG, Barreto ML, do Carmo Leal M, Monteiro CA, Schmidt MI, Paim J, et al. Health conditions and health-policy innovations in Brazil: the way forward. Lancet 2011; 377:2042-53.

32. Mathers CD, Ma Fat D, Inoue M, Rao C, Lopez AD. Counting the dead and what they died from: an assessment of the global status of cause of death data. Bull Word Health Organ 2005; 83:171-9.

33. Joubert J, Rao C, Bradshaw D, Vos T, Lopez AD. Evaluating the quality of national mortality statistics from civil registration in South Africa, 19972007. PLoS One 2013; 8:e64592.

34. Frias PG, Pereira PMH, Andrade CLT, Szwarcwald CL. Sistema de Informações sobre Mortalidade: estudo de caso em municípios com precariedade dos dados. Cad Saúde Pública 2008; 24:2257-66.

35. Pereira CCB, Vidal SA, Carvalho PI, Frias PG. Avaliação da implantação do Sistema de Informações sobre Nascidos Vivos (SINASC) em Pernambuco. Rev Bras Saúde Matern Infant 2013; 13:39-49.

36. AbouZahr C, Rampatige R, Lopez A, de Savigny D. When civil registration is inadequate: interim methods for generating vital statistics. Pac Health Dialog 2012; 18:215-30.

37. Santana M, Aquino R, Medina MG. Effect of the family health strategy on surveillance of infant mortality. Rev Saúde Pública 2012; 46:59-67.

Recebido em 19/Nov/2013

Versão final reapresentada em 06/Fev/2014

Aprovado em 27/Mar/2014 